Colloids and Surfaces

A: Physicochemical and Engineering Aspects 167 (2000) 21-30

\title{
Modeling self-assembly of surfactants at solid-liquid interfaces. II. hydrophilic surfaces
}

\author{
R.A. Johnson, R. Nagarajan * \\ Department of Chemical Engineering, The Pennsylvania State University, 161 Fenske Laboratory, University Park, \\ PA 16802-4400, USA
}

\begin{abstract}
The self-assembly of cationic and nonionic surfactants on hydrophilic surfaces is considered. By computing the equilibrium free-energy for the formation of a given structure, the critical surfactant concentration for the formation of surface aggregates (CAC) and the surface morphology are predicted. The CAC is always predicted to be lower than the bulk phase critical micelle concentration (CMC). In addition to the full spheres and cylinders that have been proposed in the literature, a series of composite structures are proposed which are composed of monolayers oriented with head groups in contact with the surface and covered with either hemispheres, hemicylinders, finite disks, or another monolayer (making the full structure a bilayer). It is shown that for several illustrative cases, composite hemicylinders will form at the CAC. Since the surfactant concentration required for the formation of full spherical and full cylindrical surface aggregates is largely comparable to the bulk $\mathrm{CMC}$, it is likely that the surface structures observed in atomic force microscopy (AFM) experiments conducted near the bulk CMC are not full spheres or cylinders, but the corresponding composite structures made up of hemispheres or hemicylinders. (C) 2000 Elsevier Science B.V. All rights reserved.
\end{abstract}

Keywords: Hydrophylic surfaces; Critical surfactant concentration; Surface aggregates; Surface morphology; Hemispheres; Hemicylinders

\section{Introduction}

Direct evidence of aggregates on hydrophilic solid surfaces has come from atomic force microscopy (AFM) [1]. Most AFM studies have focused on cationic quaternary ammonium halides on the surface of either mica or silica [2-6]. This is presumably because the surfaces of

\footnotetext{
* Corresponding author. Tel.: + 1-814-863-1973; fax: + 1814-865-7846.

E-mail address: rxn@psu.edu (R. Nagarajan)
}

mica and silica both possess a net negative charge, thus facilitating the formation of surface aggregates of cationic surfactants. Patrick et al. [6] found that, depending on the counterion, either cylinders, spheres, or lamellae form on the surface of mica. They also found that the addition of $3 \mathrm{M}$ salicylate salt induces a morphological transformation to cylinders for all cases that they investigated. Ducker and Wanless [3] studied the aggregation of dodecyl trimethyl ammonium bromide (DTAB) on the surface of mica and found that in the absence of salt, cylinders are always 
formed, as opposed to spheres forming in the bulk. Manne and Gaub [2] and Manne [1] studied the aggregation of alkyl trimethyl ammonium bromide $\left(\mathrm{C}_{n} \mathrm{TAB}\right)$ and alkyl trimethyl ammonium chloride $\left(\mathrm{C}_{n} \mathrm{TAC}\right)$ and reported observing cylindrical aggregates on mica and spherical aggregates on silica. However, for cetyl trimethyl ammonium bromide (CTAB) on the surface of mica, Ducker and Wanless [5] found that in the absence of added salt, the only stable microstructures are flat bilayers, with stable cylinders forming only upon the addition of salt. Lamont and Ducker [4] report that $\mathrm{CTA}^{+}$surfactant cations in a solution containing $\mathrm{Cs}^{+}$ions undergo a morphological transformation from bilayers to cylinders to spheres on the surface of mica upon the successive addition of $\mathrm{Cs}^{+}$ions. Considerably fewer experiments have been performed to explore the aggregation behavior of nonionic surfactants on

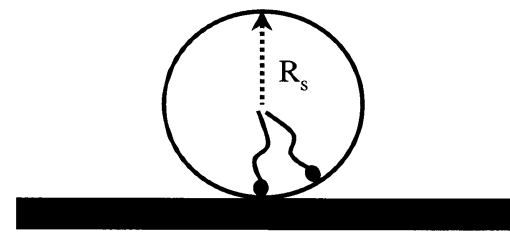

(a)

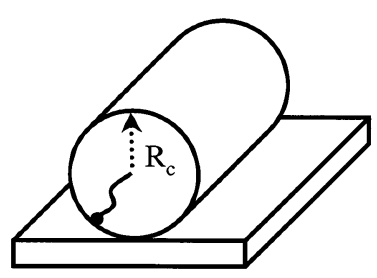

(b)

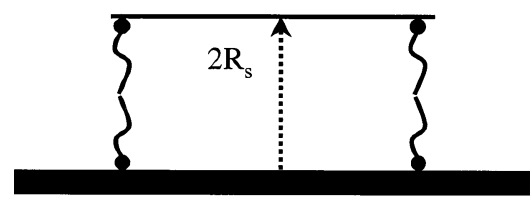

(c)

Fig. 1. Schematic representation of the structures of surfactant aggregates on a hydrophilic surface. The structures formed include (a) spherical aggregates, (b) cylindrical aggregates and (c) laterally homogeneous bilayers. hydrophilic surfaces. Nonionic ethylene oxide surfactants have been observed to form either globular aggregates or continuous bilayer structures on hydrophilic silica [7].

The experimental AFM studies mentioned above were all carried out at surfactant concentrations at or above the bulk phase critical micelle concentration (CMC) which is typically in great excess of the critical surface aggregation concentration (CAC). Accordingly, it is possible for equilibrium structures to exist on the surface at the CAC with microstructures that deviate significantly from those observed at high surfactant concentrations. To date, there has been no attempt to predict the self-assembly behavior of surfactants on a hydrophilic surface. In this paper we propose a theoretical model based on molecular thermodynamics for the self-assembly of the cationic surfactant alkyl trimethylammonium bromide $\left(\mathrm{C}_{n} \mathrm{TAB}\right)$ and the nonionic surfactant alkyl $\beta$-glucoside on hydrophilic surfaces. The theoretical approach is based on that of Nagarajan and Ruckenstein [8,9] with appropriate contributions added to account for the additional free-energy of interaction of the surfactant headgroups with the surface.

In the preceding companion paper [12] focusing on hydrophobic surfaces, the general thermodynamic relations for self-assembly, including conditions for the formation of rodlike and lamellar aggregates as well as the computational procedure employed to investigate surface aggregates are presented. Therefore, they will not be repeated here. We note that among the shapes of aggregates on hydrophilic surfaces considered are spheres, globular, and cylindrical micelles which resemble those in bulk solutions (see Fig. 1). Therefore, all the geometrical relations and freeenergy expressions formulated for bulk aggregates [9] apply also to the surface aggregates. In addition, we consider the formation of hemispheres on monolayers, hemicylinders on monolayers and bilayer lamellae. The latter structures resemble those formed on hydrophobic surfaces (discussed in the preceding paper [12]), with the hydrophobic surface generated by the monolayer in contact with the solid surface. 


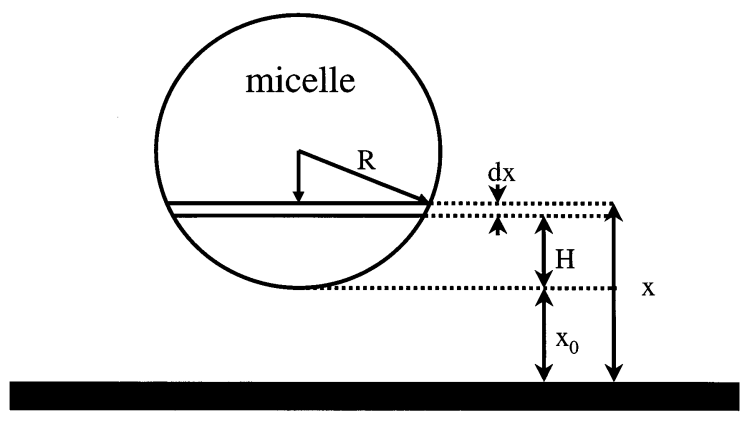

surface

Fig. 2. Schematic diagram showing the quantities needed to evaluate the interaction energy between a curved aggregate and a planar surface.

\section{Free-energy of surface aggregation}

The size distribution equation, in conjunction with the contributions to the free-energy of micellization and the geometrical properties of the aggregate allow one to calculate the equilibrium properties of the system. The standard free-energy difference $\Delta \mu_{g}^{0}$ between a surfactant molecule in an aggregate of size $g$ and one in the singly dispersed state can be decomposed into a number of contributions on the basis of molecular considerations as

$$
\begin{aligned}
\Delta \mu_{g}^{0}= & \left(\Delta \mu_{g}^{0}\right)_{\text {trans }}+\left(\Delta \mu_{g}^{0}\right)_{\text {def }}+\left(\Delta \mu_{g}^{0}\right)_{\text {inter }}+\left(\Delta \mu_{g}^{0}\right)_{\text {steric }} \\
& +\left(\Delta \mu_{g}^{0}\right)_{\text {ionic }}+\left(\Delta \mu_{g}^{0}\right)_{\text {surf }}
\end{aligned}
$$

With the exception of the contribution due to surfactant/surface interactions, $\left(\Delta \mu_{g}^{0}\right)_{\text {surf }}$, expressions for each of the above contributions to the free-energy of aggregation are summarized in $\mathrm{Na}$ garajan and Ruckenstein [9].

The surfactant/surface interactions are considered to provide two contributions. One is the electrostatic interaction between the charged surfactant head groups and the hydrophilic surface, if the surface is charged. The other is the free-energy change associated with displacing water by the surfactant head groups wherever aggregatesolid surface contact occurs. An expression for the surfactant-surface electrostatic interaction free-energy is developed via the solution of Poisson's equation for the specific geometry in question. The two surfaces (i.e. the aggregate and the solid surface) can have different charge densities. Three aggregate geometries are considered taking the solid surface to be planar: two charged planes interacting (which represents lamellar aggregate structures), a cylinder interacting with a plane and a sphere interacting with a plane. The free-energy expression for all three cases is derived based on the well-known solution for two interacting planes with dissimilar charge densities [10]. Consider two planar surfaces, with charge densities $\sigma_{1}$ and $\sigma_{2}$ and separated by a distance $x$, then

$$
\begin{aligned}
E(x)= & \frac{\epsilon \kappa}{2}\left[\left(\Psi_{1}^{2}+\Psi_{2}^{2}\right)(1-\operatorname{coth} \kappa x)\right. \\
& \left.+2 \Psi_{1} \Psi_{2} \operatorname{csch} \kappa x\right]
\end{aligned}
$$

where $E(x)$ is interaction energy per unit area of surface, $\epsilon$ is the dielectric constant of the solvent, $\kappa$ is the reciprocal Debye length and

$$
\begin{aligned}
& \Psi_{1}=\frac{\sigma_{1}}{\epsilon \kappa} \operatorname{csch} \kappa x+\frac{\sigma_{2}}{\epsilon \kappa} \operatorname{coth} \kappa x \\
& \Psi_{2}=\frac{\sigma_{1}}{\epsilon \kappa} \operatorname{coth} \kappa x+\frac{\sigma_{2}}{\epsilon \kappa} \operatorname{csch} \kappa x
\end{aligned}
$$

In order to approximate the interaction between the curved geometries representing the aggregates and the planar surface, an approximate representation for the above equations is desired. This can be accomplished by approximating the hyperbolic functions by their appropriate series expansion. In this case, both $\operatorname{coth}(z)$ and $\operatorname{csch}(z)$ can be approximated to leading order by $1 / z$, which is strictly valid for $z \ll 1$. Thus, Eq. (2) takes on the approximate form

$E(x)=\frac{\left(\sigma_{1}+\sigma_{2}\right)^{2}}{\varepsilon \kappa}\left[\frac{1}{\kappa x}\right]^{2}$

To calculate the interaction for a curved geometry with the planar surface, one simply integrates the planar solution over the surface area of the aggregate following the Deryaguin method [11]

$E_{\mathrm{tot}}=\int E(x) \mathrm{d} A$

where the differential area element $\mathrm{d} A$ will be a function of the specific geometry of the aggregate and $E_{\text {tot }}$ is the total interaction energy. If the aggregate is assumed to be spherical (see Fig. 2), then 
$\mathrm{d} A=2 \pi \sqrt{R^{2}-(R-H)^{2}} \mathrm{~d} x$

where $H=\left(x-x_{0}\right), R$ is the radius of the aggregate at which the charge is located and $x_{0}$ is the distance of separation between the charge on the aggregate and the solid surface (see Fig. 2). Thus, Eq. (6) becomes

$$
\begin{aligned}
E_{\mathrm{tot}}= & \frac{2 \pi\left(\sigma_{1}+\sigma_{2}\right)^{2}}{\epsilon \kappa^{3}} \int_{x_{0}}^{x_{0}+2 R}\left[\frac{1}{x}\right]^{2}\left[R^{2}\right. \\
& \left.-\left(R-x+x_{0}\right)^{2}\right]^{1 / 2} \mathrm{~d} x
\end{aligned}
$$

We numerically evaluate the integral in Eq. (8) using Gaussian quadrature, then approximate the functional form using a non-linear LevenbergMarquardt regression. A comparison of the regression to the numerically evaluated integral is shown in Fig. 3. Thus, Eq. (8) becomes

$E_{\mathrm{tot}}=\frac{2 \pi\left(\sigma_{1}+\sigma_{2}\right)^{2}}{\epsilon \kappa^{3}} I\left(R ; x_{0}\right)$

where

$$
\begin{aligned}
& I\left(R ; x_{0}\right) \\
& =\exp \left[-0.635-1.062 \ln \left(\frac{x_{0}}{R}+0.02969\right)\right]
\end{aligned}
$$

We now use the above procedure to evaluate the interaction energy between a cylindrical surface aggregate and a planar surface, noting that

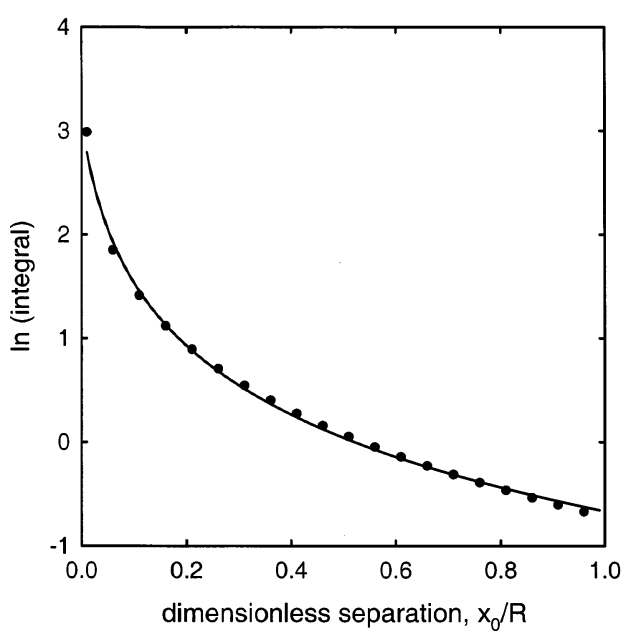

Fig. 3. Comparison of the regression Eq. (10) to the numerical evaluation of the integral in Eq. (8). the integral that results can be evaluated analytically. For this case the differential area element is taken to be $\mathrm{d} A=2 L \mathrm{~d} x$ where $L$ is the length of the cylinder. Thus, the interaction energy per unit length of cylinder becomes

$\frac{E_{\mathrm{tot}}}{L}=\frac{2\left(\sigma_{1}+\sigma_{2}\right)^{2}}{\epsilon \kappa}\left[\frac{1}{\kappa^{2}}\left(\frac{1}{x_{0}}-\frac{1}{x_{0}+2 R}\right)\right]$

The interaction energy per unit surface area for two planar surfaces with dissimilar charges is evaluated in the same manner as

$\frac{E_{\mathrm{tot}}}{A}=\frac{\left(\sigma_{1}+\sigma_{2}\right)^{2}}{\epsilon \kappa}\left[\frac{1}{\kappa x_{0}}\right]^{2}$

Finally, the free-energy of interaction (per $k T$ ) is evaluated. The surface charge density of the surfactant aggregate, $\sigma_{1}$, is taken to be $\sigma_{1}=e / a_{\mathrm{s}}$ where $e$ is the electronic charge and $a_{\mathrm{s}}$ is the aggregate-water contact per molecule. For all three cases, the energy $E_{\text {tot }}$ is normalized by the total surface area of the aggregate, then multiplied by the area per molecule for surfactant headgroups to put the free energy on a molecular basis. Thus, for spherical aggregates

$\frac{\left(\Delta \mu_{\mathrm{g}}^{0}\right)_{\text {surf,el }}}{k T}=\frac{a_{\mathrm{s}}\left(\frac{e}{a_{\mathrm{s}}}+\sigma_{2}\right)^{2}}{2 R^{2} k T \epsilon \kappa^{3}} I\left(R ; x_{0}\right)$

for cylindrical aggregates

$\frac{\left(\Delta \mu_{g}^{0}\right)_{\text {surf,el }}}{k T}=\frac{a_{\mathrm{s}}\left(\frac{e}{a_{\mathrm{s}}}+\sigma_{2}\right)^{2}}{\pi R k T \epsilon \kappa}\left[\frac{1}{\kappa^{2}}\left(\frac{1}{x_{0}}-\frac{1}{x_{0}+2 R}\right)\right]$

and for planar aggregates

$\frac{\left(\Delta \mu_{\mathrm{g}}^{0}\right)_{\text {surf,el }}}{k T}=\frac{a_{\text {head }}\left(\frac{e}{a_{\mathrm{s}}}+\sigma_{2}\right)^{2}}{k T \epsilon \kappa}\left(\frac{1}{\kappa x_{0}}\right)^{2}$

where $a_{\text {head }}$ is the surface area of the aggregate per molecule that is in contact with the solid surface and $a_{\text {head }}=a_{\mathrm{s}} / g$ for spheres and cylinders, $a_{\text {head }}=$ $a_{\mathrm{s}} / 2$ for bilayers and $a_{\text {head }}=a_{\mathrm{s}}$ for monolayers.

The second contribution associated with surfactant-solid surface interactions is that due to the displacement of water by the aggregate head groups at the solid surface. This contribution is always relevent irrespective of whether the surface 
Table 1

Molecular constants for surfactant head groups

\begin{tabular}{llll}
\hline Surfactant head group & $a_{p}\left(\mathrm{~nm}^{2}\right)$ & $a_{0}\left(\mathrm{~nm}^{2}\right)$ & $\delta(\mathrm{nm})$ \\
\hline $\begin{array}{l}\text { Alkyl trimethylammonium } \\
\text { bromide }\end{array}$ & 0.54 & 0.21 & 0.345 \\
$\beta$-Glucoside & 0.40 & 0.21 & 0.0 \\
\hline
\end{tabular}

is charged or not. If these interactions between the head groups and the solid surface are more favorable compared to the solid surface-water interactions, there will be a negative contribution to the free-energy of aggregation. This contribution can be calculated by considering the formation of a new aggregate head group-solid surface interface replacing the water-solid surface interface, as

$\frac{\left(\Delta \mu_{g}^{0}\right)_{\text {surf,int }}}{k T}=-\frac{\gamma}{k T} a_{\text {head }}$

The displacement tension $\gamma$ can be viewed as the difference between the water-solid surface interfacial tension $\gamma_{\text {surf }-\mathrm{w}}$ and the aggregate head group-solid surface interfacial tension, $\gamma_{\text {surf-agg. }}$. The latter tension can represent various types of interactions involving uncharged surfaces, such as dipolar interactions, hydrogen bonding interactions, hydration interactions, etc. Note that $\gamma$ in this case is likely to be smaller than the displacement tension for a hydrophobic surface, which can be on the order of 50 dynes $\mathrm{cm}^{-1}$ [12]. Thus, the total contribution of hydrophilic surface/surfactant interactions to the free energy of surface aggregation is

$\left(\Delta \mu_{\mathrm{g}}^{0}\right)_{\text {surf,tot }}=\left(\Delta \mu_{\mathrm{g}}^{0}\right)_{\text {surf,int }}+\left(\Delta \mu_{\mathrm{g}}^{0}\right)_{\text {surf,el }}$

For the case of ionic surfactants, the electrostatic contribution to $\left(\Delta \mu_{\mathrm{g}}^{0}\right)_{\text {surf,tot }}$ will dominate. On the other hand, for nonionic surfactants $\left(\Delta \mu_{\mathrm{g}}^{0}\right)_{\text {surf,el }}$ will vanish and the only driving force for the formation of surface micelles is $\left(\Delta \mu_{\mathrm{g}}^{0}\right)_{\text {surf,int. }}$ As for bulk-phase surfactant micellization, the transfer free-energy is large, negative and independent of the micellar size, and is thus primarily responsible for the aggregated state being favored over the singly dispersed state. The contribution due to the surface discussed above is also negative. Thus, the surface aggre- gated state is favored over the bulk aggregated state.

\subsection{Estimation of molecular constants}

The hydrophobic portion of the surfactants considered are composed of aliphatic hydrocarbon chains. The associated molecular constants are the volume, $v_{\mathrm{s}}$ and the extended length, $l_{\mathrm{s}}$ of the tail. The required molecular constants for the head group are the cross-sectional area, $a_{\mathrm{p}}$ and the surface area per molecule shielded from contact with solvent by the head group, $a_{0}$ for all types and the distance, $\delta$ from the core surface to where the counterion is located for ionic types. It should be emphasized that all molecular constants are derived from the molecular structure of the surfactant, and are not adjustable parameters. The molecular constants are estimated as in reference [9] and are summarized in Table 1 for each type of surfactant head group under consideration.

The solid surface is considered to be characterized by two material constants, namely $\gamma$, the displacement tension, and $\sigma_{2}$, the surface charge density. For all calculations presented here, the displacement tension has been fixed at a representative value of 10 dynes $\mathrm{cm}^{-1}$. The surface charge density depends on whether the surface is one of constant charge, or one of constant potential. For the case of a surface of constant charge, the nature of the charge depends on the internal structure of the solid and is independent of the concentration of ions in the surrounding fluid phase. On the other hand, for a surface of constant potential, an equilibrium exists between the ions in solution and those making up the surface. Thus, the surface charge is a function of the concentration of potential-determining ions in the bulk. The dependence of the surface charge density, $\sigma_{2}$, for a surface of constant potential follows directly from a solution to the Poisson-Boltzmann equation for the total charge density in the diffuse double-layer, which must equal the surface charge density in order to satisfy electrical neutrality [10]. After integration of the point charge density over the entire double-layer, the Grahame equation results 
$\sigma_{2}=0.731$ charges $\mathrm{nm}^{-2} C^{1 / 2} \sin \mathrm{h}\left(\frac{\Psi_{2}}{51.4 \mathrm{mV}}\right)$

where $C$ is the molar concentration of added electolyte in the solution. Eq. (18) is valid for a symmetric electrolyte in water at $25^{\circ} \mathrm{C}$. For the surfaces of atomically smooth mica and silica, the surface potentials are estimated as in Manne [1], resulting in the following dependence of surface charge density on electrolyte concentration:

$\sigma_{2} \begin{cases}\left(3.7 \text { charge } \mathrm{nm}^{-2}\right)[\text { electrolyte }]^{1 / 2} & \text { mica } \\ \left(1.7 \text { charge } \mathrm{nm}^{-2}\right)[\text { electrolyte }]^{1 / 2} & \text { silica }\end{cases}$

where [electrolyte] is the ionic strength in molar units taken to be $0.1 \mathrm{M}$ in all calculations. Finally, the spacing $x_{0}$ between the aggregate and the solid surface has been fixed at $0.5 \mathrm{~nm}$. This value was chosen to be on the order of the size of a typical surfactant headgroup. It should be noted that the above set of material constants were merely chosen to represent typical hydrophilic surfaces for simulation purposes and were not chosen with reference to any specific experimental measurement.

\section{Results and discussion}

\subsection{General aggregation behavior}

If spheres, cylinders and bilayers are considered as the possible equilibrium shapes, as suggested in the literature, our calculations show that both ionic and nonionic surfactants would self-assemble as laterally homogeneous bilayers on hydrophilic surfaces at the CAC. Fully formed spherical and cylindrical aggregates are never favored as equilibrium surface structures at the CAC. For an illustrative value of $n_{c}=12$, the CAC values corresponding to bilayers (normalized by the corresponding bulk $\mathrm{CMC}$ ) are $\mathrm{CAC} / \mathrm{CMC}=0.00089$ and $\mathrm{CAC} / \mathrm{CMC}=0.012$ for cationic $\mathrm{CTAB}$ on mica and silica, respectively and $\mathrm{CAC} / \mathrm{CMC}=$ 0.16 for nonionic $\beta$-glucoside. Since the ratio $\mathrm{CAC} / \mathrm{CMC}$ is always less than unity, surface aggregation is preferred to bulk aggregation. In contrast to the behavior predicted for aggregation on hydrophobic surfaces [12], the ratio CAC/ $\mathrm{CMC}$ for nonionic surfactants is roughly an order of magnitude larger for aggregation on hydrophilic surfaces. The reason for this is that on hydrophobic surfaces, the driving force for the aggregation of nonionic surfactants is a tendency of aggregates to shield the surface from exposure to solvent represented by a large displacement tension of $\gamma=50$ dynes $\mathrm{cm}^{-1}$. On the other hand, for hydrophilic surfaces, aggregation is driven by specific hydrogen bonding and van der Waals attraction between the surface and the surfactant head groups, which is represented by smaller magnitudes for the displacement tension $\gamma$ (which is taken to be 10 dynes $\mathrm{cm}^{-1}$ in the present calculation). For ionic $\mathrm{CTAB}$, the $\mathrm{CAC} / \mathrm{CMC}$ ratio is much smaller compared to the nonionic surfactant because of the favorable electrostatic contributions provided by the aggregate/surface interaction. Further, since the surface charge density of mica is taken to be larger than that of silica, the $\mathrm{CAC} / \mathrm{CMC}$ ratio on mica is correspondingly smaller than that on silica.

Although no results have been reported in the literature, it is interesting to consider the aggregation of anionic surfactants such as sodium alkyl sulfates, on the same negatively charged hydrophilic surfaces. Although the electrostatic interaction between the surface and the surfactant head groups will be repulsive, aggregation can still be possible due to the displacement tension effect. However, the specific interactions between the surfactant head group and the surface must be strong enough to overcome the electrostatic repulsion. This balance is only possible for surfaces with sufficiently small charge densities. For example, anionic sodium dodecyl sulfate (SDS) is predicted to form globular micelles in dilute electrolyte solutions. However, surface aggregation is predicted to take place only when $\gamma \geq 45$ dynes $\mathrm{cm}^{-1}$ and on surfaces of extremely low charge density, i.e. $\sigma_{2} \geq 0.04$ charge $\mathrm{nm}^{-2}$. The driving force for surface aggregation is the favorable interaction between the surfactant head group and the surface, which is a function of the contact area between the surfactant and the surface, a head. However, this contact area is very small for spherical and cylindrical surface aggregates, be- 
cause their geometry allows limited contact with a planar surface. Thus, in order for this contribution to the total free-energy of aggregation to be appreciable, the magnitude of the displacement tension must be sufficiently large.

As mentioned in the introduction, AFM experiments have shown that structures such as full spheres, full cylinders and bilayers are possible on hydrophilic surfaces, whereas predictions based on the theory presented above indicate the formation of only bilayers at the CAC. The model predictions are not surprising since the surface aggregation is driven primarily by the aggregatesurface interactions, which is maximized for the lamellar structure that possesses the largest contact area per molecule, $a_{\text {head }}$. Given this fact, how is it possible that spherical or cylindrical surface aggregates (for which the surfactant head group-

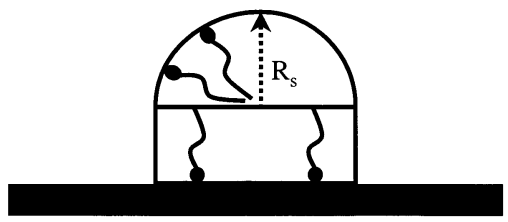

(a)

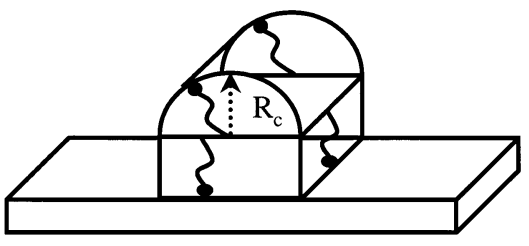

(b)

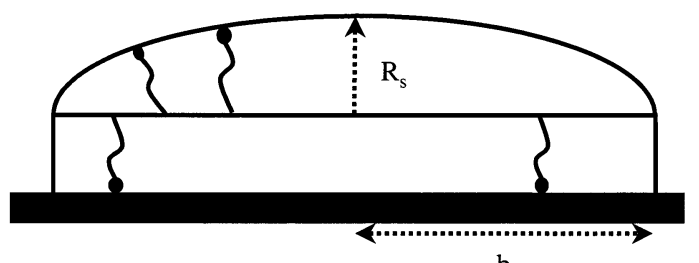

(c)

Fig. 4. Schematic representation of composite structures of surfactant aggregates on a hydrophilic surface. Each structure is composed of a monolayer oriented with the surfactant head groups in contact with the surface as the base, topped with hemispheres (a), hemicylinders (b), and a second monolayer with opposite orientation (c). solid surface contact area are very small) are observed? To answer this question we note that the microstructures reported from AFM experiments are at least partially based on conjucture since the AFM images are only capable of revealing the topology of the surfactant/solution interface and the distance from that interface to the solid surface. In view of this, we propose new surface structures (see Fig. 4) which yield identical surface profiles (viewed from above as in an AFM experiment) to those reported in the literature. Common to all of these structures is a surfactant monolayer oriented such that the head groups are in contact with the hydrophilic surface. The formation of this structure by itself involves the creation of a hydrophobic aggregate core-water contact and this is highly unfavorable. However, this interface is identical to that of a hydrophobic surface discussed in the preceding paper [12] with displacement tension $\gamma=50$ dynes $\mathrm{cm}^{-1}$. Thus, it is postulated that the structures described in [12] can form on the hydrophobic surface presented by the monolayer, in effect shielding any contact between the hydrophobic tails and water. As a result, we obtain the postulated composite structures consisting of a monolayer on the solid surface above which are hemispheres, hemicylinders, or a second monolayer (i.e. a bilayer).

The free-energy for the formation of the composite structures shown in Fig. 4 can be calculated by combining the free-energies of formation for the monolayer base with that for the top portion made up of hemispherical, hemicylindrical, or monolayer aggregates. The free-energy per molecule of the structures on the top layer are computed as for aggregates on hydrophobic surfaces, discussed in the preceding paper [12]. The free-energy of formation of the monolayer base is estimated as for infinite monolayers, with the exception that the formation of the surfactant core-water interface is not included since it will be fully covered by the aggregates on the top. The free-energies per molecule for the composite aggregates are then calculated by dividing the total free-energy by the total number of molecules in the composite structures. The free-energies for the formation of the various composite aggregates shown in Fig. 4 are predicted to be lower than 
Table 2

Surface aggregation behavior on hydrophilic surfaces

\begin{tabular}{|c|c|c|c|}
\hline \multicolumn{4}{|c|}{ (a) AFM experimental results } \\
\hline Quaternary ammonium & low salt & cylinders and bilayers & {$[1-3,5,6]$} \\
\hline Halides/mica & high salt & spheres & [4] \\
\hline $\begin{array}{l}\text { Quaternary ammonium } \\
\text { Halides/silica }\end{array}$ & - & spheres & {$[1,2]$} \\
\hline $\mathrm{C}_{n} \mathrm{E}_{x} /$ silica & - & globules and bilayers & [7] \\
\hline \multicolumn{4}{|c|}{ (b) Model predictions of microstructures at $C A C$} \\
\hline Surfactant & Bulk microstructure & Surface microstructure & \\
\hline $\mathrm{C}_{12} \mathrm{TAB} / \mathrm{mica}$ & spheres & hemicylinders on monolayers & \\
\hline $\mathrm{C}_{12} \mathrm{TAB} /$ silica & spheres & hemicylinders on monolayers & \\
\hline$\beta$-Glucoside & cylinders & hemicylinders on monolayers & \\
\hline
\end{tabular}

those for the formation of the full structures shown in Fig. 1 (see Tables 3A and 3B). Thus, the composite structures with hemispheres or hemicylinders on the top layer fully covering the monolayer underneath are energetically more favorable than the full spheres or cylinders. The general aggregation behavior for cationic $\mathrm{C}_{12} \mathrm{TAB}$ and nonionic $\beta$-Glucoside with $n_{c}=12$ predicted by this approach is summarized in Table 2 . For both cationic $\mathrm{C}_{12} \mathrm{TAB}$ and nonionic $\beta$-Glucosides, composite hemicylinders are formed at the CAC. Indeed, the full spheres and full cylinders suggested by the AFM studies are possible only when the total surfactant concentration is very large as discussed below. For these conditions, the alternate possibility of composite structures also exists, but with the hemispheres or hemicylinders not fully covering the monolayers underneath. Therefore, it is quite possible that these full structures never form and the AFM observations are those of the composite structures proposed here.

\subsection{Influence of surfactant concentration}

As outlined in the preceding paper [12], since the surface area of solids in a typical AFM experiment is small, an increase in the total surfactant concentration (over the CAC) implies an increase in $X_{1}$ and thus in the chemical potential of the surfactant. In this manner, free-energy barriers for the formation of a certain type of aggregate can be overcome by an increase in the concentration of singly dispersed surfactant, $X_{1}$. The progression of free energies are shown in Table $3 \mathrm{~A}$ for some

Table 3A

Minimum free-energy values for aggregates on hydrophilic surfaces with $\gamma=10$ dynes $\mathrm{cm}^{-1}$

\begin{tabular}{|c|c|c|c|c|}
\hline & & $\mathrm{C}_{12} \mathrm{TAB}$ & & $\beta$-glucoside \\
\hline \multirow{5}{*}{ Full structures } & Microstructure & $\Delta \mu_{g}^{0}(\mathrm{kT}): \operatorname{mica}^{\mathrm{a}}$ & $\Delta \mu_{g}^{0}(\mathrm{kT}):$ silica & $\Delta \mu_{g}^{0}$ \\
\hline & bilayer & -16.5 & -13.96 & -14.5 \\
\hline & sphere & -10.3 & -9.80 & -12.6 \\
\hline & cylinder (endcap) & -9.11 & -8.49 & -11.3 \\
\hline & cylinder (middle) & -10.6 & -9.98 & -13.1 \\
\hline \multirow[t]{4}{*}{ Composite structures } & hemisphere & -17.8 & -15.74 & -15.9 \\
\hline & hemicylinder (endcap) & -18.3 & -16.1 & -16.1 \\
\hline & hemicylinder (middle) & -19.1 & -16.69 & -16.7 \\
\hline & finite disk & -16.2 & -14.6 & -16.22 \\
\hline
\end{tabular}

\footnotetext{
a The mica and silica surfaces are distinguished from one another by differing surface charge densities, as discussed in the text.
} This distinction is not relevent in the case of nonionic surfactants. 
illustrative cases. The free-energies for the formation of composite structures (patterned on a monolayer oriented with head groups in contact with the solid surface) are lower than those for full structures on a hydrophilic surface. For the composite structures, immediate surface saturation on the top layer is a requisite condition since any aggregate core-water contact is forbidden. For the illustrative case of $\mathrm{C}_{12} \mathrm{TAB}$ on the hydrophilic mica surface, the progression of surface microstructures is shown in Fig. 5. It is clear that the addition of even a small amount of surfactant (changing $X_{1}$ from the CAC to $2.2 \times \mathrm{CAC}$ ) can induce a morphological transformation between the composite structures. Once the bilayer is

(a)
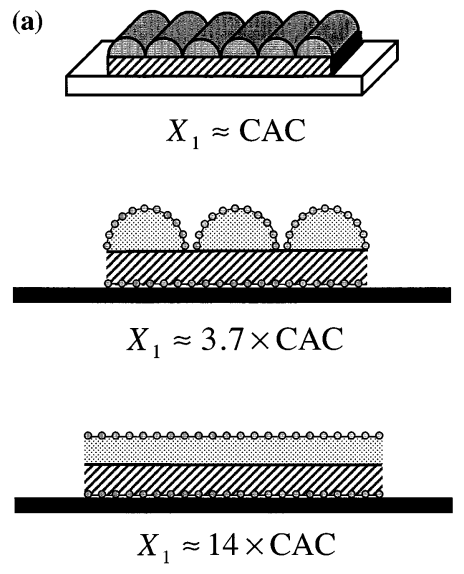

(b)

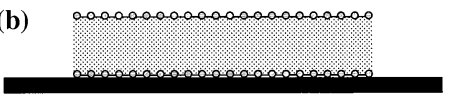

$$
X_{1} \approx 14 \times \mathrm{CAC}
$$

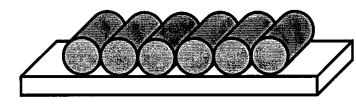

$$
X_{1} \approx 4900 \times \mathrm{CAC}
$$

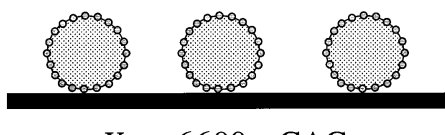

$$
X_{1} \approx 6600 \times \mathrm{CAC}
$$

Fig. 5. Progression of aggregate microstructures as the concentration of singly dispersed ionic surfactant, $X_{1}$, is increased beyond the CAC.
Table 3B

Proposed concentration-dependent change based on the above free-energy estimates ${ }^{\mathrm{a}}$

\begin{tabular}{cll}
\hline Ionic on mica & Ionic on silica & Nonionic \\
\hline Composite hemi- & Composite hemi- & Composite hemi- \\
cylinder & cylinder & cylinder \\
$\left(X_{1}=\right.$ CAC $)$ & $\left(X_{1}=\right.$ CAC $)$ & $\left(X_{1}=\right.$ CAC $)$ \\
$\downarrow$ & $\downarrow$ & $\downarrow$ \\
Composite hemi- & Composite hemi- & Composite finite \\
sphere & sphere & disk \\
$\left(X_{1} \sim 3.7\right.$ CAC $)$ & $\left(X_{1} \sim 2.6\right.$ CAC $)$ & $\left(X_{1} \sim 1.6\right.$ CAC $)$ \\
$\downarrow$ & $\downarrow$ & $\downarrow$ \\
Bilayer & Composite finite & Composite hemi- \\
& disk & sphere \\
$\left(X_{1} \sim 14\right.$ CAC $)$ & $\left(X_{1} \sim 8.1\right.$ CAC $)$ & $\left(X_{1} \sim 2.2\right.$ CAC $)$ \\
$\downarrow$ & $\downarrow$ & $\downarrow$ \\
Composite finite & Bilayer & Bilayer \\
disk & & \\
$\left(X_{1} \sim 18\right.$ CAC) & $\left(X_{1} \sim 15\right.$ CAC $)$ & $\left(X_{1} \sim 9.0\right.$ CAC $)$ \\
$\downarrow$ & $\downarrow$ & $\downarrow$ \\
Full cylinder & Full cylinder & Full cylinder \\
$\left(X_{1} \sim 4900\right.$ CAC $)$ & $\left(X_{1} \sim 820\right.$ CAC $)$ & $\left(X_{1} \sim 37\right.$ CAC $)$ \\
$\downarrow$ & $\downarrow$ & $\downarrow$ \\
Full sphere & Full sphere & Full sphere \\
$\left(X_{1} \sim 6600\right.$ CAC) & $\left(X_{1} \sim 980\right.$ CAC $)$ & $\left(X_{1} \sim 60\right.$ CAC $)$ \\
\hline & & \\
\hline & &
\end{tabular}

${ }^{\text {a }}$ valid for $n_{c}=12$ and $\gamma=10$ dynes $\mathrm{cm}^{-1}$.

formed, $X_{1}$ has to increase by a large magnitude if full spheres $\left(X_{1} \simeq 6600 \times\right.$ CAC) or full cylinders $\left(X_{1} \simeq 4900 \times \mathrm{CAC}\right)$ are to form. For such large $X_{1}$, it is also possible for the composite structures to exist with the hemispheres or hemicylinders not fully covering the monolayer underneath. Thus, a transformation from the composite structures to the full structures is unlikely to take place for this ionic surfactant on mica or on silica. For nonionic surfactant, the increase in CAC that is necessary for a shape transformation to full spheres or full cylinders is much smaller than that for ionic surfactants (see Table 3B). However, even for the nonionic surfactants the composite structures are unlikely to transform into full spheres or cylinders. Fig. 5 and Tables $3 \mathrm{~A}$ and $3 \mathrm{~B}$ demonstrate the wide range of surface aggregate structures that are possible as functions of surfactant concentration in a typical AFM experiment. 


\section{Conclusions}

A theory of surfactant self-assembly at the interface between a hydrophilic solid surface and the solvent is developed that depends on only the molecular properties of the surfactant in question and the properties of the surface. The theory extends the well established treatment of surfactant self-assembly in solution, with a new contribution to the free-energy of aggregation that accounts for the attraction between the hydrophilic surface and the surfactant headgroups. Two parameters are needed to fully characterize the surface/surfactant interactions: the charge density, $\sigma_{2}$, of the solid surface and the displacement tension, $\gamma$, which can be viewed as the difference between the water-solid surface interfacial tension $\gamma_{\text {surf }- \text { w }}$ and the aggregate head groupsolid surface interfacial tension, $\gamma_{\text {surf }- \text { agg. }}$.

Model predictions are made for cationic alkyl trimethylammonium bromide and nonionic alkyl $\beta$-glucoside surfactants. Due to the attraction between surfactant head groups and hydrophilic surfaces, the critical condition for self-assembly at the surface (the CAC) is always lower than the bulk $\mathrm{CMC}$ and is typically more than an order of magnitude lower than the bulk CMC. Considering full spheres, full cylinders and bilayers as the only allowed surface aggregate structures, laterally homogenous bilayers are always predicted to form at the CAC. This is in contrast to the suggested spherical, cylindrical and lamellar surface aggregates inferred via AFM experiments. To explain the difference between the predicted and observed surface structures, composite structures patterned on the hydrophobic surface presented by a monolayer with the surfactant head groups in contact with the solid surface are proposed. In this manner, composite hemicylindrical surface aggregates of cationic and nonionic surfactants are predicted to form at the CAC. As the surfactant concentration is increased, it is possible for surface aggregates to undergo a morphological transformation to other surface microstructures. Since the surfactant concentration required for the formation of full spherical and full cylindrical surface aggregates is large, comparable to the bulk CMC, it is more likely that the surface structures observed in AFM experiments conducted near the bulk CMC are not spheres or full cylinders, but the corresponding composite structures made up of hemispheres or hemicylinders.

\section{Acknowledgements}

We acknowledge the interesting AFM studies of Professors Ducker and Manne which stimulated this theoretical study. Conversations with Professor Ducker in the context of model development are gratefully acknowledged.

\section{References}

[1] S. Manne, Progr. Colloid Polym. Sci. 103 (1997) 226-233.

[2] S. Manne, H.E. Gaub, Science 270 (1995) 1480-1482.

[3] W.A. Ducker, E.J. Wanless, Langmuir 12 (1996) 59155919.

[4] R.E. Lamont, W.A. Ducker, J. Am. Chem. Soc. 120 (1998) 7602-7607.

[5] W.A. Ducker, E.J. Wanless, Langmuir 15 (1999) 160168.

[6] R. Nagarajan, Colloids and Surfaces A 71 (1993) 39-64.

[7] L.M. Grant, F. Tiberg, W.A. Ducker, J. Phys. Chem. B 102 (1998) 4288-4294.

[8] H. Shinto, S. Tsuji, M. Miyahara, K. Higashitani, Langmuir 15 (1999) 578-586.

[9] R. Nagarajan, E. Ruckenstein, Langmuir 7 (1991) 2934 2969.

[10] R.J. Hunter, Foundations of Colloid Science, vol. 1, Oxford University Press, New York, 1987, p. 412.

[11] B.V. Deryaguin, Discuss. Faraday Soc. 18 (1954) 85.

[12] R.A. Johnson, R. Nagarajan, Colloids and Surfaces A, preceding paper. 